\title{
ACTIVE SPACE DEBRIS REMOVAL BY USING LASER PROPULSION
}

\author{
Yu. A. Rezunkov \\ Public Corporation "Research Institute \\ for Optic-Electronic Instrument Engineering" (NII OEP) \\ Sosnovy Bor, Leningrad Region 188540, Russia
}

\begin{abstract}
At present, a few projects on the space debris removal by using highpower lasers are developed. One of the established projects is the ORION proposed by Claude Phipps from Photonics Associates Company and supported by NASA (USA) [1]. But the technical feasibility of the concept is limited by sizes of the debris objects (from 1 to $10 \mathrm{~cm}$ ) because of a small thrust impulse generated at the laser ablation of the debris materials. At the same time, the removal of rocket upper stages and satellites, which have reached the end of their lives, has been carried out only in a very small number of cases and most of them remain on the Low Earth Orbits (LEO). To reduce the amount of these large-size objects, designing of space systems allowing deorbiting upper rocket stages and removing large-size satellite remnants from economically and scientifically useful orbits to disposal ones is considered. The suggested system is based on high-power laser propulsion. Laser-Orbital Transfer Vehicle (LOTV) with the developed aerospace laser propulsion engine is considered as applied to the problem of mitigation of man-made large-size space debris in LEO.
\end{abstract}

\section{INTRODUCTION}

It is known that the near-Earth space is widely explored starting from the first launch of sputnik into the LEO in October 4, 1957. This onrush exploration of the space resulted in the growth of such problem as space debris occupying the LEO space. In accordance with an express information published by the Russian Academy of Sciences [2], there are about 23,000 objects, whose sizes exceed $10 \mathrm{~cm}$, and about 8,600 objects, whose mass is more than $2 \mathrm{~kg}$, on the LEO. Monitoring of the near-Earth space revealed also that there are 54 nuclear power reactors, looping the Earth. Most of the reactors were used as power supply units of satellites, which completed their orbital life. The nuclear power units are usually removed from LEO to Geostationary Earth Orbits (GEO) to

This is an Open Access article distributed under the terms of the Creative Commons Attribution License 2.0, which permits unrestricted use, distribution, and reproduction in any medium, provided the original work is properly cited. 
stave off their falling to the Earth. Nevertheless, for the safety reason, the reactors have to be transferred to longer distances from the Earth, for example, to a libration point (region) in the Earth-Moon system [2].

The safety standard of space flights developed in NASA (NSS 1740.14) states that the rocket stages, satellites, and other orbital objects should be removed out of the LEO after completing their missions by using one of the following ways [3]:

- maneuvering missions into a storage orbit for subsequent conversion;

- controlled aerocapture to return the satellite into a permitted region of the Earth; and

- transferring to the orbit with a limited ballistic life period of the satellite (time duration of the passive ballistic period does not exceed 25 years because of the atmosphere drag).

At present, there have been developed a few techniques, both passive and active, of removing the large-size objects from the LEO onto the Earth or to other orbits.

The use of electrodynamic cables arranged on a satellite board is one of the examples of the passive technique. There are two ways of the cable application to remove LEO satellites. In the first one, the cable module is fastened to a satellite before its launching. After finishing of the satellite mission, the modulus unrolls conductive belt with the length of hundreds of meters. Gradient gravity force stretches the belt along the local vertical line so that the belt not only increases the aerodynamic resistance of the satellite but also produces the electrodynamic resistance due to a passive interaction of the conductive cable with the Earth electromagnetic field. The total effect of the forces provides the satellite removal with a 9-ton mass from a 1000-kilometer orbit in 25 years. The second version assumes removing of satellites via a preliminary capture of the debris objects by using large conductive nets and then removing it with a cable of a 30-kilometer length. It should be noted that the cable systems have one principal demerit consisting in uncontrolled returning of the satellites into the Earth atmosphere.

Solar sails can also be used to remove large-size debris objects out of the Earth orbits. The sails are usually arranged onboard of satellites or rocket upper stages, and they unfold after completing of the equipment missions. Also, in this case, deorbiting of a 500-kilogram object from a 700-kilometer orbit is assumed to take 25 years. But there is a great chance of such satellites to collide with other space debris objects, in which the sail itself can be destroyed.

The use of active removal systems based on orbital tug-boats seems to be a more attractive way of removal of large-size debris from the Earth orbits [2]. The tug-boats have to be incorporated into new space transportation systems. Such system has to include both a few inter-orbital tug-boats and storing waste satellites on GEO in specially designed load assembly units transferring the assembly 
units to the return orbit. For example, Energia, a Russian space corporation [4], has developed a special space "tug" which relies on a nuclear power unit. The unit is supposed to be completed in design and testing by 2020, and to be in service no later than 2023. It will have a long mission life span and a nuclear power source point in addition to its drive technique. Radio thermal space waves can generate electricity over a long time, making an ideal power source for ion drives, which use electric fields to accelerate ionized gas, as one of the versions of the microwave propulsion. Similar technology could be used on a larger scale in the "interceptor" spacecraft. But this tug system is expected to have a big size and mass to be launched into the LEO. Moreover, the system per se will represent a danger for the Earth.

The principal advantage of the laser propulsion $[5,6]$ as compared to other thrust production systems is the use of the power of remote lasers to produce a thrust, which allows decreasing of the fuel margin and increasing of the payload mass of a space tug. Laser orbital transfer vehicle tug is based on the remote propulsion system, using the power of remote lasers arranged on the Earth or in the space [6]. In accordance with the LOTV concept, to transfer the LOTV tug with the mass of $10 \mathrm{t}$ from LEO to GEO in a short period of time, minimal thrust of hundred of newtons has to be produced [7]. A preliminary technology assessment shows that the laser power of $500 \mathrm{~kW}$ is required to produce the thrust like this. The required laser power depends also on a particular design of an on-board laser propulsion engine (LPE), a propellant composition, and laser propulsion mechanisms used.

In this paper, the LOTV tug is considered as a high-power laser propulsion system, including the following units: $(i)$ an LPE operating by a specific mechanism of the laser propulsion production; (ii) a vehicle with the LPE and an optical receiver system on board; and (iii) a ground or space-borne laser with a transmitter telescope system.

At consideration of the LOTV concept, the efficiency of the laser power delivery to the space tug has to be taken into account, including the limits imposed by the receiver optics collecting the laser power. A preliminary design of laser thrusters is conceptualized too. Capturing of space debris objects and attaching them onto the LOTV board should be considered separately because there are a few capture techniques developed around the world at the present time.

\section{LASER PROPULSION FOR SPACE MISSIONS}

The laser propulsion is based on the following mechanisms of laser radiation interaction with a propellant, namely: $(i)$ the laser breakdown of gases and the generation of blast waves (or shock waves); (ii)the laser ablation of solid propellants; and ( $i i i)$ the laser detonation of power-generating materials due to the energy release of an internal energy of the material under the laser power. 
The laser breakdown of gases is considered usually as applied to launching of satellites in the atmosphere when the air can be used as a propellant to produce a thrust [6]. The laser ablation propulsion can be applied to satellite space missions because a specific impulse more than $5000 \mathrm{~s}$ is achieved in the case when heatresisting materials are ablated under a high-peak power laser pulse [8]. The use of power-generating materials has to increase the laser propulsion efficiency in both cases of launching and space missions of satellites.

To characterize the thrust production efficiency, such parameter as a momentum coupling coefficient is used. The coefficient is determined as the ratio of the thrust $T$ to the laser power $P$, i. e., $C_{m}=T / P$. This parameter determines the minimal laser power to produce a 1-newton thrust. The propellant consumption in LPE is characterized by the specific impulse $I_{\mathrm{sp}}$, which is equal to the ratio of the thrust to the propellant consumption per second, $\dot{m},: I_{\mathrm{sp}}=T /(\dot{m} g)=v / g$, where $v$ is the average exhaust velocity and $g$ is the acceleration of gravity. Both thrust parameters define the total efficiency $\eta$ of the laser propulsion as follows [5]:

$$
\eta=\frac{C_{m} I_{\mathrm{sp}} g}{2}
$$

The physical and technical limits of the laser propulsion in terms of various mechanisms of laser radiation interaction with propellants are considered in [9]. Here, the general attention is paid to the laser ablation propulsion, including the ablation of power-generating materials, because this type of thrust propulsion is more adapted to LOTV space missions [8].

The laser ablation includes three different processes:

(1) a direct laser ablation of solid propellants when the propulsion is produced due to the pressure of an evaporated material (the evaporation mechanism of the propulsion production);

(2) a combined ablation when a shock wave due to the laser breakdown of an evaporated material closely by its surface is originated; and

(3) the laser detonation of power-generating propellants, including $\mathrm{CHO}$ polymers.

It is shown experimentally that the momentum coupling coefficient $C_{m}$ of the laser propulsion under the direct laser ablation depends on the type of the propellant and laser pulse characteristics. There are different estimates of the maximal $C_{m}$ in the developed laser ablation propulsion theory. According to [8], the upper limit of $C_{m}$ for the specified material is achieved with rather a long laser pulse of $\sim 1 \mathrm{~ms}$, and it weakly depends on the laser radiation wavelength:

$$
C_{m \max }=\frac{A_{2}}{a} \frac{\gamma T_{b}}{m_{i}}
$$


Here, $A_{2}$ is the absorption coefficient of the laser power by a solid material during its evaporation; $a$ is the specific heat of evaporation $(\mathrm{J} / \mathrm{g}) ; \gamma$ is the effective adiabatic index of the evaporated material; $T_{b}$ is the material boiling point; and $m_{i}$ is the mass of atoms or molecules of the evaporated material. Resulting from the formula, to get the maximal $C_{m}$, it is necessary to choose a material with the maximal ratio $\gamma T_{b} / a^{2} m_{i}$, and to provide the maximal absorption of the laser power by the material. Estimations of $C_{m}$ coincide with the experimental data on $C_{m}$ closely, for example: $C_{m} \max =6 \cdot 10^{-5} \mathrm{~N} / \mathrm{W}$ for aluminum and $10 \cdot 10^{-5} \mathrm{~N} / \mathrm{W}$ for cooper.

But ignition of plasma close to the surface of a solid material by a laser pulse when the laser radiation intensity exceeds the laser-breakdown threshold causes $C_{m}$ to decrease. In this case [8], the following self-similar approximation for the momentum coupling coefficient is performed with a good accuracy if the laser radiation intensity exceeds the breakdown threshold of the evaporated material:

$$
C_{m}=b\left(I \lambda \tau^{1 / 2}\right)^{n} .
$$

Here, $I$ is the radiation intensity $\left(\mathrm{W} / \mathrm{cm}^{2}\right) ; \lambda$ is the radiation wavelength $(\mathrm{cm})$; $\tau$ is the pulse duration (s); $b$ and $n$ are the empirical parameters, for example, $b=5.6$ for aluminum alloys and 6.5 for $\mathrm{C}-\mathrm{H}$ materials, and $n=-0.300$ for both materials. The ratio (1) is a regular one over a wide range of the laser pulse intensity variations from $3 \mathrm{MW} / \mathrm{cm}^{2}$ to $70 \mathrm{TW} / \mathrm{cm}^{2}$, the pulse duration from $500 \mathrm{ps}$ to $1.5 \mathrm{~ms}$, and the radiation wavelength from 0.248 to $10.6 \mu \mathrm{m}$. It is significant that the formula (1) makes it possible to generalize the examination of different types of lasers for the laser propulsion but at some restrictions given below.

Characteristic variable $I \lambda \tau^{1 / 2}$ appears in the theory of laser ablation [8] as a parameter similarity, used to describe the interaction of a laser pulse with solid materials. In the theory, it is assumed that $(i)$ the material strongly absorbs the laser radiation and the absorption depth exceeds the value of $1 / \mu_{\lambda}$, where $\mu_{\lambda}$ is the absorption coefficient at the specified radiation wavelength $\lambda$; and (ii) the radiation intensity on a solid surface corresponds to the conditions of the production of the maximal specific recoil impulse in a vacuum if the radiation is not screened by the originated plasma. It is also assumed that the laser-induced plasma absorbs the radiation owing to the inverse Bremsstrahlung effect.

The coefficient $C_{m}$ obtained experimentally under the direct laser ablation for many metals and nature materials does not exceed tens of dyn $/ \mathrm{W}\left(10^{-5} \mathrm{~N} / \mathrm{W}\right)$. One of the possible ways of increasing $C_{m}$ is producing an additional thrust by shock waves, generated under a laser breakdown of evaporated materials close to the solid surface. At that, the laser plasma extension is accompanied by strong shock waves increasing the gas pressure behind it up to $10^{3}-10^{6}$ atm and the plasma temperature up to tens or hundreds of electron volts. In vacuum, the effect results in increase of $C_{m}$ by 1-2 orders as compared with the direct laser ablation. 
In the author's opinion, significant increase in the laser propulsion efficiency can be attained by using energetic propellants of CHO-chemical composition, in which the laser pulse initiates chemical reactions with a noticeable chemical energy release. To find out the influence of energy characteristics of the CHOmaterials upon the production efficiency of the laser propulsion, a number of polymeric and polycrystalline materials of $\mathrm{CHO}$ compositions with the negative oxygen balance [10] was studied including the specific heat of the combustion, the detonation, and the delayed burning of detonation products. It is known [11] that the laser radiation induces evaporation and the thermal decomposition of the CHO-materials. This is a pyrolysis starting at the temperature of 500$700{ }^{\circ} \mathrm{C}$ in the absence of oxygen. In air, the reaction of the high-temperature oxidation (combustion) of the pyrolysis products results in the energy release via two successive chemical reactions, namely, the detonation and the delayed burning of detonation products: $Q=Q_{\mathrm{det}}+N Q_{\mathrm{db}}$, where $N$ is the coefficient of afterburning of detonation products. The energy of the detonation and delayed burning reactions may be calculated by the Hess law [11]:

$$
Q_{\mathrm{det}}=-\left(\Delta H_{\mathrm{pr}}-\Delta H_{f 298}^{o}\right) ; \quad Q_{\mathrm{db}}=-\Delta H_{\mathrm{pr}}
$$

where $\Delta H_{\mathrm{pr}}$ is the enthalpy of the reaction products; and $\Delta H_{f 298}^{o}$ is the enthalpy of the material.

The energy balance of vapors of CHO-materials and the combustion of its components in oxygen of the air, which corresponds to standard conditions of laboratory experiments at the laser ablation of the materials, can be expressed in the following form:

$$
(M+m) \frac{v^{2}}{2}=\beta[\alpha E+m Q]
$$

where $m$ is the mass of the evaporated material; $M$ is the air mass in a volume of the laser propulsion nozzle; $v$ is the average exhaust velocity; $\alpha$ is the coefficient of the laser energy conversion into heat energy of the propellant; and $\beta$ is the coefficient of the conversion of propellant heat energy into the exhaust flow kinetic energy.

Then in accordance with the general definition of the momentum coupling coefficient $C_{m}$, one has:

$$
\begin{aligned}
& C_{m 1}=\frac{J}{E}=\frac{M v}{E}=\sqrt{\frac{2 M \beta \alpha}{E}} ; \\
& C_{m 2}=\frac{J}{E}=\frac{(M+m) v}{E}=\sqrt{\frac{2(M+m) \beta[\alpha E+m Q]}{E^{2}}}
\end{aligned}
$$

where $C_{m 1}$ is the momentum coupling coefficient of the laser air-breathing propulsion under atmospheric conditions; $C_{m 2}$ is the momentum coupling coefficient with the $\mathrm{CHO}$-materials as the auxiliary propellants. The relative increase 
$K$ (an efficient factor) of the momentum coupling coefficient can be defined in the case of the use of the laser detonation as

$$
K=\frac{C_{m 2}}{C_{m 1}}=\sqrt{\left(1+\frac{m}{M}\right)\left(1+\frac{m Q}{\alpha E}\right)} .
$$

To analyze the thrust impulse production in LPE with CHO-chemical solid propellants, the parameter of specific ablation energy $Q^{*}=E / m[8]$ can be used in addition. Then one consequently has:

$$
K=\frac{C_{m 2}}{C_{m 1}}=\sqrt{\left(1+\frac{m}{M}\right)\left(1+\frac{Q}{\alpha Q^{*}}\right)} .
$$

Figure 1 demonstrates the experimental results for $C_{m}$ as a function of $K$ $\sim\left[1+2.5\left(Q_{\mathrm{det}} / Q^{*}+N Q_{\mathrm{db}} / Q^{*}\right)\right]^{1 / 2}$ for polymeric propellants $(1)$ over the range $1.75 \leq K \leq 3.12$ under atmospheric conditions without regard to the presence of oxygen in the polymer composition. Diagram 2 corresponds to the dependence of $C_{m}$ upon $K$ for polycrystalline propellants, the values of which for polyoxymethylene $(N=1)$, metaldehyde, and carbamoyl hydrazine $(N>1)$ are proportional over the range $3.08 \leq K \leq 5.96$. The values of $C_{m}$ vs. $K$ for dihydroxybenzene and oxybenzoic acid $(N<1)$ have a large spread and do not correspond to the dependence of $C_{m}$ on $K$.

The experiments showed that the delayed burning of detonation products of the evaporated CHO-materials under the laser detonation in oxygen of the air is similar for all of the materials. And the reaction is the oxidation of the released atomic carbon and gaseous hydrogen with a formation of carbon dioxide and water molecules. At that, the delayed burning takes place sequentially to the detonation reaction and is limited by the bounded content of oxygen in the atmosphere air.

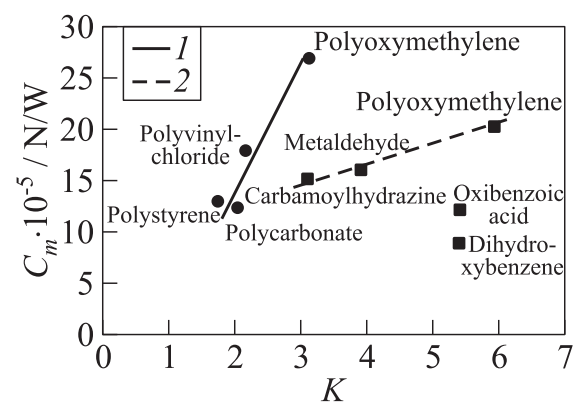

Figure 1 Dependence of $C_{m}$ on $K$ for polymeric (1) and polycrystalline (2) CHO-type propellants at atmosphere conditions [10] 
Analysis of the experimental data on the laser propulsion with the polymeric materials used in the form of a plane target [10] shows that the maximal $C_{m}=27 \cdot 10^{-5} \mathrm{~N} / \mathrm{W}$ relates to polyoxymethylene, an oxygen-containing linear polymer with a high detonation component in the momentum coupling coefficient. The polymer has a pronounced region of the $\mathrm{CO}_{2}$-laser radiation absorption by the $\mathrm{C}-\mathrm{O}-\mathrm{C}$ stretching vibrations.

The experiments show also that the efficient factor $K$ can be applied to selecting of the polymer propellants for laser propulsion. For example, a moderate $K$ is achieved for polymers with high $Q^{*}(5,000-50,000 \mathrm{~kJ} / \mathrm{kg})$ that agrees well with the sufficiently high experimental values of $C_{m}$. In the case of the polycrystalline materials the values of $Q^{* *}$ are significantly lower $(1200-4000 \mathrm{~kJ} / \mathrm{kg}$ ) that leads to a noticeable increase of $K$ (two times for polyoxymethylene). But $C_{m}$ for polycrystalline propellants is nevertheless 2.5 times less than the forecast increase. At that, for both polymeric and polycrystalline propellants their own proportional dependence of $C_{m}$ upon $K$ (for $N \geq 1$ ) is observed.

As one can see, the use of only laser ablation technique to produce a thrust does not allow to sufficiently increase the production efficiency by laser power. To reach a higher laser propulsion efficiency, the author suggests to use a combined mechanism of the thrust production when the laser ablation of solid (or liquid) propellants is realized inside a combustion chamber and special device is used to form an impulse as conventional jet engines produce.

In this case, the total efficiency of laser propulsion with energetic materials can be written as a relation of the power of an exhaust jet to the total power of heat sources in a combustion chamber of LPE in the following manner:

$$
\eta_{\mathrm{LPE}}=\frac{\beta(\alpha+m Q / E)}{1+m Q / E} .
$$

The results on $\eta_{\text {LPE }}$ with Delrin ${ }^{\circledR}$ and polymethylmethacrylate (PMMA), studied in the experiments and re-calculated for space and atmosphere conditions, are listed in Table 1.

Atmosphere conditions of the thrust production mean the condition of delayed burning of the combustion products in the presence of the atmosphere

Table 1 Efficiency $\eta_{\mathrm{LPE}}$ of the experimentally tested CHO-materials $(\lambda=10.6 \mu \mathrm{m}$; $\alpha=0.4 ;$ and $\beta=0.9$ )

\begin{tabular}{lrccc}
\hline CHO-material & $E, \mathrm{~J}$ & $\dot{m} Q_{\mathrm{det}}, \mathrm{J}$ & $\dot{m} Q_{\mathrm{det}} / E$ & $\begin{array}{c}\eta_{\mathrm{LPE}}, \\
\text { space/atmosphere conditions }\end{array}$ \\
\hline Delrin $^{\circledR}{ }^{\circledR}[10]$ & 61.0 & 33.19 & 0.54 & $0.55 / 0.81$ \\
Delrin $^{\circledR}[10]$ & 57.1 & 21.60 & 0.38 & $0.51 / 0.78$ \\
PMMA [12] & 90.0 & 18.51 & 0.21 & $0.45 / 0.86$ \\
PMMA [12] & 130.0 & 19.73 & 0.15 & $0.43 / 0.85$ \\
\hline
\end{tabular}


oxygen. As one can see, a sufficient increase in the efficiency of laser propulsion is observed in the case when a complete propellant burning is achieved. To satisfy this condition, a space vehicle should imply both a CHO-propellant and auxiliary oxygen onboard. In accordance with the chemical reactions following the laser detonation of CHO-polymers, it will require about $1.067 \mathrm{~kg}$ (746.7 l) of oxygen for $1 \mathrm{~kg}$ of $\left(\mathrm{CH}_{2} \mathrm{O}\right)_{n}$.

\section{TECHNICAL FEATURES OF LASER ORBITAL TRANSFER VEHICLE}

A LOTV vehicle is considered as a space tug-boat for transferring large-size debris objects from LEO to GEO or into a "libration point" in the Earth-Moon system. That is why the vehicle is expected to have a large inertial mass about $10 \mathrm{t}$. To provide a continuous mode of the thrust production independently of atmosphere conditions, an air-born laser with a power of $500 \mathrm{~kW}$ is assumed to be used (Fig. 2) [6]. In this case, the produced thrust of $100 \mathrm{~N}$ is much less than the gravitational forces pressing to the vehicle by the Earth. In this case one says about a low-thrust maneuver mode of the vehicle mission from orbit to orbit by a spiral trajectory [13], and the orbital transferring time depends on both the vehicle mass and the laser propulsion efficiency.

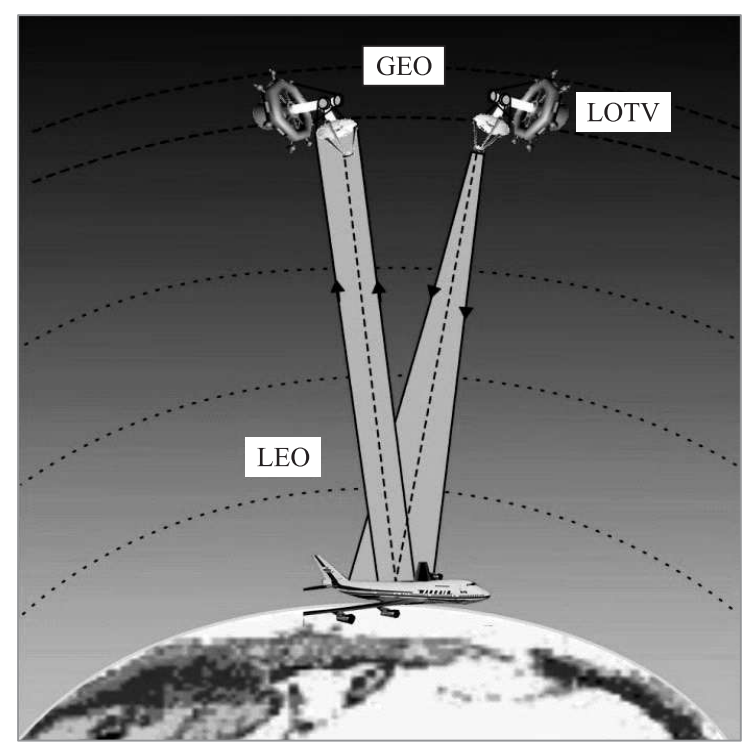

Figure 2 The LOTV ("space tugboat") concept. LOTV mass $M_{0} \approx 10$ t; operating cycle $\sim 1$ month; and airborne laser power $\approx 500 \mathrm{~kW}$ 
To define the laser energy consumption for the LOTV orbital mission, such parameter as expenditure of the laser energy $C_{e}[7]$ was applied to transfer a unit mass (kilogram) of the LOTV payload up to a high orbit. In the analysis, the following conditions of the LOTV mission have been taken into consideration:

- transferring of LOTV is run with a variable vehicle mass;

- specific impulse $I_{\mathrm{sp}}$ of LPE is an optimal one satisfying the conditions of high production efficiency of laser propulsion; and

- the energy cost of the orbital LOTV maneuver is a function of both the LOTV receiver aperture and the LPE thrust and specific impulse.

To analyze the space maneuver of LOTV, the transfer scenario of the vehicle within a coplanar trajectory was considered. The vehicle has the initial velocity $V_{\mathrm{LEO}}$ and mass $M_{0}$. Moreover, the fact that the LOTV receiver mirror collecting incoming laser beam intercepts only a portion of the laser power because the mirror is limited by its aperture was taken into account. In the diffraction approximation, the portion of the laser power collected by the mirror comes in terms of spherical Bessel function $J_{0}$ as follows:

$$
\eta_{\mathrm{dif}}=1-J_{0}^{2}\left(\frac{\pi}{2} \frac{d_{\mathrm{em}} d_{\mathrm{rec}}}{\lambda\left(r-R_{\text {Earth }}\right)}\right)-J_{1}^{2}\left(\frac{\pi}{2} \frac{d_{\mathrm{em}} d_{\mathrm{rec}}}{\lambda\left(r-R_{\text {Earth }}\right)}\right)
$$

where $R_{\text {Earth }}$ is the Earth radius; $d_{\mathrm{em}}$ and $d_{\text {rec }}$ are the diameters of the transmitter and receiver mirrors; and $r$ is the current orbit radius. The use of airborne lasers imposes also some limitations on the aperture of the laser transmitter telescope. The transmitter mirror diameter will most likely be not more than $1.5 \mathrm{~m}$.

The total mass of the LOTV is taken to be equal to the sum of the initial mass of the vehicle $M_{0}$ and the receiver mirror mass $M_{\text {mir }}$. The initial mass $M_{0}$ includes the debris and the propellant masses. The present-day technology used for manufacturing of large-aperture space mirrors gives a distributed mass of the receiver of $1-5 \mathrm{~kg} / \mathrm{m}^{2}$ [14]. That means the mass of a 30 -meter receiver can run up to $3 \mathrm{t}$. This is quite comparable with $M_{0}(10 \mathrm{t})$, and it will increase the period of the LOTV space mission and the mission energy expenditure. It is suggested the LPE mass is negligibly small as compared with the LOTV mass. The efficiency of the conversion of the laser power into kinetic power of the exhaust jet $\eta$ is chosen to be constant and equal to 0.6 .

In the LOTV maneuver model, it is assumed that the vehicle has the velocity $\overrightarrow{\mathbf{V}}$ at start moment and the momentum impulse $\overrightarrow{\mathbf{p}}_{\text {in }}=(M+\Delta m) \overrightarrow{\mathbf{V}}$. The propellant consumption mass $\Delta m$ is ejected at a velocity $\overrightarrow{\mathbf{V}}_{E}$ in the vehicle spatial value system. As a result, the total final impulse of the space vehicle and of the propellant mass will be equal to $\overline{\mathbf{p}}_{\text {fin }}=M(\overline{\mathbf{V}}+\overline{\Delta \mathbf{V}})+\Delta m\left(\overline{\mathbf{V}}+\overline{\mathbf{V}}_{E}\right)$ 
Table 2 The LOTV mission from LEO to a libration point of the Earth-Moon system $\left(C_{m}=12.23 \cdot 10^{-5} \mathrm{~N} / \mathrm{W} ;\right.$ thrust $=122.36 \mathrm{~N} ;$ and propellant consumption $\left.=12.48 \mathrm{~g} / \mathrm{s}\right)$

\begin{tabular}{|c|c|c|c|c|}
\hline $\begin{array}{c}\text { LOTV initial mass } \\
M_{0}, \mathrm{t} \\
\text { (two versions) }\end{array}$ & $\begin{array}{l}\text { LOTV receiver } \\
\text { mirror diameter } \\
D, \mathrm{~m}\end{array}$ & $\begin{array}{c}\text { Payload mass } \\
\text { delivered to } \\
\text { a libration point } \\
M_{\mathrm{pl}}, \mathrm{t}\end{array}$ & $\begin{array}{c}\text { Total } \\
\text { mission } \\
\text { period, } \\
24 \mathrm{~h}\end{array}$ & $\begin{array}{c}\text { Specific energy } \\
\text { consumption } C_{e} \\
\mathrm{MJ} / \mathrm{kg}\end{array}$ \\
\hline $10.39 / 100.39$ & 10 & $1.75 / 32.4$ & $6.3 / 48.8$ & $309 / 130$ \\
\hline $11.57 / 101.57$ & 20 & $1.11 / 31.8$ & $6.9 / 49.35$ & $535 / 134$ \\
\hline $13.534 / 103.534$ & 30 & $0.52 / 30.8$ & $7.84 / 50.3$ & $1305 / 141$ \\
\hline
\end{tabular}

in a stationary coordinate system, and increment in $\overrightarrow{\Delta \mathbf{p}}$ is described by the formula:

$$
\overrightarrow{\Delta \mathbf{p}}=M \overrightarrow{\Delta \mathbf{V}}+\Delta m \overrightarrow{\mathbf{V}}_{E} .
$$

Taking into account that $\Delta m=-\Delta M$ and using the Newton's second law of motion $d \overrightarrow{\mathbf{p}} / d t=\overrightarrow{\mathbf{F}}$, one can get [13]:

$$
\frac{d \overline{\mathbf{p}}}{d t}=M \frac{d \overline{\mathrm{V}}}{d t}-\frac{d M}{d t} \overline{\mathrm{V}}_{E}=-\gamma \frac{M_{\mathrm{Earth}} M}{r^{3}} \overline{\mathbf{r}}
$$

or

$$
\frac{d \overrightarrow{\mathbf{V}}}{d t}-\frac{\overrightarrow{\mathbf{V}}_{E}}{M} \frac{d M}{d t}=-g \frac{R_{\text {Earth }}^{2}}{r^{3}} \overrightarrow{\mathbf{r}} .
$$

The calculations made by using (2) and (3) show that the minimum of the energy expenditure per $1 \mathrm{~kg}$ of payload $C_{e}$ increases with the increase of the specific impulse. And the optimal diameter of the receiver mirror constitutes $D_{\mathrm{LOTV}} \sim 17 \mathrm{~m}, C_{e}$ comes to $1.6 \cdot 10^{8} \mathrm{~J} / \mathrm{kg}$ for $\lambda=1.06 \mu \mathrm{m}$. The estimated receiver diameter is a crucial point in many respects, if the atmosphere effects on the efficiency of the laser power delivery to the vehicle through the upper atmosphere are to be examined. One should be mentioned that the specific energy consumption by using the solid fuel inter-orbital tug IUS (Inertial Upper Stage) comes to $C_{e}=5 \cdot 10^{7} \mathrm{~J} / \mathrm{kg}$, and it comes to $2 \cdot 10^{8} \mathrm{~J} / \mathrm{kg}$ for the mission with a liquid fuel rocket of the "Centaurus" type [15]. But the main advantage of the LOTV space maneuver in respect to the vehicles with a conventional chemical thrust is that the LOTV does not bear an energy supply source on a board.

Similar theoretical estimations of the transfer characteristics of the LOTV from the LEO to the Earth-Moon libration point are listed in Table 2. The LOTV vehicle with two different masses $M_{0}=10$ and $100 \mathrm{t}$ are considered assuming different masses of the debris objects which have to be removed from the LEO in the course of one mission.

As one can see, the smaller is the mirror diameter, the larger is the payload mass delivered to a libration point. The last outcome is particularly evident in 
the case of the LOTV with a low initial mass. And only 6 days will require to transfer the LOTV to the libration point in this case. Nevertheless, one may note that the debris inertial mass in the LEO can be reduced to $30 \mathrm{t}$ only during one mission if the LOTV has the primary mirror diameter of $30 \mathrm{~m}$ and the initial mass of $100 \mathrm{t}$.

\section{LASER PROPULSION ENGINE FOR SPACE MISSIONS AND LASER THRUSTER FOR SATELLITES}

The limited receiver aperture of LOTV results both in decreasing of the laser power delivered to the propulsion engine being on high orbits exceeding $5000 \mathrm{~km}$ and in decreasing of the thrust produced by LPE as a consequence. The lowthrust mode of the vehicle flight implies a spiral trajectory of the flight when the spiral orbit is close to a circular orbit. It means that the axis of the laser beam coming to the vehicle does not coincide with the vehicle movement vector, and auxiliary optics have to be applied to direct the laser beam into LPE, providing a freedom of the LOTV orbit maneuvers on a mutual orientation of the vehicle and laser.

For that, it is assumed [6] to use an auxiliary space reflector which would direct the laser beam into the engine nozzle. The reflector has to be arranged in the vehicle orbit or near it and should maneuver in the orbit by the same manner as the vehicle. Moreover, a laser beam directed into LPE from the nozzle exhaust side would be distorted by the exhaust jet. And the combination of the optical and gas-dynamic units as a common unit of the engine $[6,8]$ would result in a degradation of the optical surface of the nozzle under a load of extremely high temperature plasma, plasma electrons, and the ion flux.

To design the LPE for LOTV, a different approach to designing the engine was used. The approach is based on the constructive and functional separation of the engine optical and nozzle units. The conceptual design of Aerospace LPE (ASLPE) [16] is shown in Fig. 3. Each unit can be separately designed to fully meet the specific characteristics of lasers and to provide given thrust characteristics for specific conditions of the ASLPE operation.

Aerospace LPE operates as follows. A beam from a remote laser passes to the optical receiver system (a radiation collector and additional optical elements) arranged on the vehicle board. The receiver system transforms the laser beam and directs it coaxially to the symmetry axis into the LPE beam concentrator.

The laser beam formed by the optical system is transmitted to the first reflector $\mathrm{R} 1$, which is made as a cone-shaped figure of rotation with a generatrix being a part of a parabola. The focus of the mirror R1 is a circle (shown in the figure as a dashed ellipse line), which crosses the plane of the figure in the 


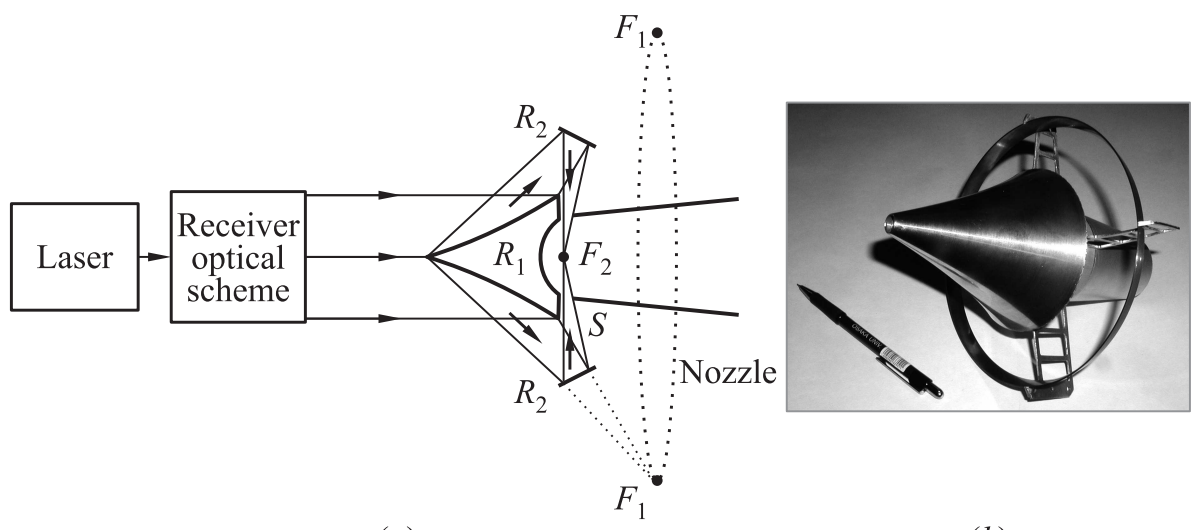

(a)

(b)

Figure 3 Conceptual design $(a)$ and model photo $(b)$ of ASLPE

point $F_{1}$. The second reflector $R_{2}$ changes the position of the beam focus point so that the beam is directed into the nozzle. Generally, the form and sizes of focus region $F_{2}$ can change, depending on the shape of generatrix of the reflector $R_{2}$. When the optical parameters of both reflectors are selected, the laser beam is be focused inside the nozzle in such a way that the radiation intensity is great enough to induce a laser breakdown of a solid propellant being arranged closely by the nozzle axis.

The engine nozzle consists of two components - a shock wave impulse receiver and the jet nozzle itself. The pressure impulse receiver and the first reflector are parts of the combined element that is a center-body of the engine. The form and geometry parameters of the pressure impulse receiver are determined at considering an optimum arrangement of the propellant breakdown region about its walls in approach of a theory of local explosion.

There are no optical windows for the radiation input into a nozzle in the ASLPE device. And the gas window can be produced by a boundary of the flow inside the nozzle with an external atmosphere. In this case, an extra external nozzle producing a directed gas flow and an additional thrust is mounted downwards the flow from the engine beam concentrator. Thus, the total effect of two nozzles should result in keeping the specific impulse of the exhaust jet as a whole in a repetitively pulsed mode of laser operation.

The prototype of the aerospace laser propulsion engine (Fig. $3 b$ ) was designed and experimentally tested by using a $\mathrm{CO}_{2}$ laser operating in a repetitively pulsed mode with the pulse energy of $130 \mathrm{~J}$ and the pulse repetition rate of $50 \mathrm{~Hz}$, the laser output power was of $6 \mathrm{~kW}$ [17]. The total mass of the model is $150 \mathrm{~g}$. Polyoximethylene (Delrin ${ }^{\circledR}$ ) was used as auxiliary propellant to increase a thrust in the experiments. The ASLPE model demonstrated the following laser propulsion characteristics: 


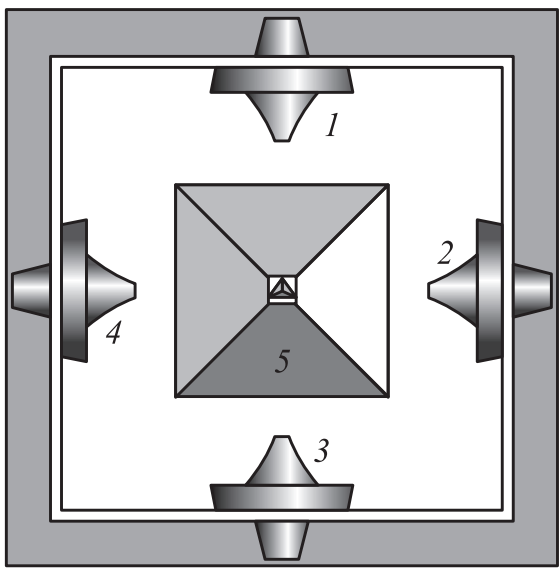

Figure 4 Concept of the laser fine-adjustment thrusters on the base of ASLPE

- momentum coupling coefficient $C_{m}=2.5 \cdot 10^{-4} \mathrm{~N} / \mathrm{W}$; and

- thrust $T=1.3-1.5 \mathrm{~N}$.

Another model of the ASLPE was tested with other $\mathrm{CO}_{2}$ laser operating in continuous wave $(\mathrm{CW})$ modes. In the experiments, the maximal thrust achieved was $2.0 \mathrm{~N}$ under the laser power of $10 \mathrm{~kW}$ and at the auxiliary air mass flow of $0.2 \mathrm{~g} / \mathrm{s}$. The thrust corresponds to $C_{m}=10^{-4} \mathrm{~N} / \mathrm{W}$.

The suggested device of ASLPE can be easily employed to design a space satellite laser thruster, to arrange on a satellite board till its launching. The thruster is schematically shown in Fig. 4. The thruster combines a four pair wise orthogonal ASLPE (1-4) arranged on an integrated platform. In the center of the platform, a tetrahedral mirror pyramid (5) is installed to direct a laser beam to one (or pair) engines.

A more detailed design of the thruster is shown in Fig. 5 to illustrate how the device can operate. Two possible versions of the propellant storage are shown in the figure. Liquid fuel tanks can be arranged on a platform closely by every engine to inject the fuel into a laser breakdown region of the engine nozzle. Simultaneously, solid propellants can also be installed at the engine axis to decrease the laser intensity threshold initiating the laser ablation process of the propellant.

A reference $\mathrm{He}-\mathrm{Ne}$ laser is used to stabilize a spatial position of the laser power beam in a plane of the thruster receiver mirror (or rhomb). This operational requirement is caused by the vibration of a satellite construction and by the influence of its motion on the high-power laser radiation delivery to the thruster. To control the optical part of the thruster and operating laser beam, 


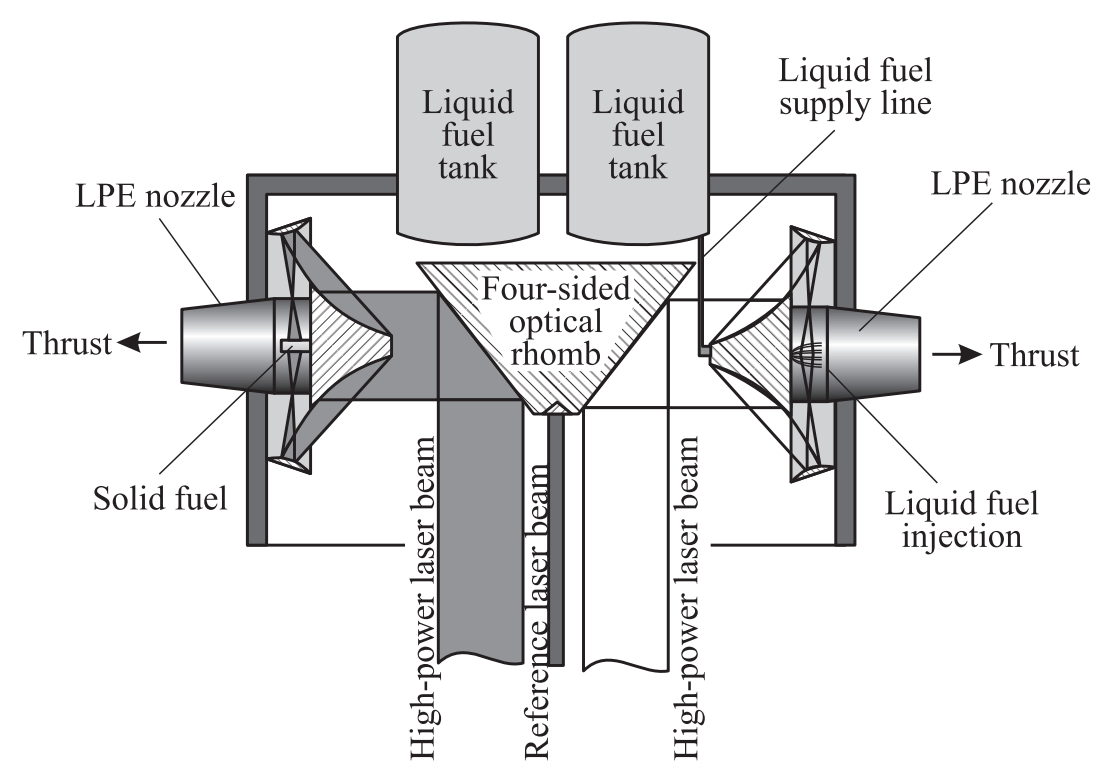

Figure 5 Principal schematic diagram of the laser thruster designed on a basis of ASLPE [18]

the adjustment system is supplied with a laser illumination system by using auxiliary adjusting lasers and retroreflectors arranged on the top of the optical rhomb.

The thruster can be used to control the satellite operational orbit or to remove rocket upper stages back to the Earth. To realize this technique, the thruster has to be arranged onboard of the rocket stage and need only be switched on such a moment of time when the stage moves above a fixed local point of the Earth. The LPE can operate individually or by pairs, producing various directions of the thrust vector depending on the satellite orientation with respect to the orbital motion vector.

The satellite orbit correction or its deorbiting by using the suggested laser thruster can be estimated theoretically by considering the satellite orbital motion as the movement of its center of inertia. In this case, the satellite is considered as a point mass being under the influence of various disturbing forces [18]. The results of the estimations are presented in Table 3, where the following parameters are listed:

- $m_{\mathrm{pl}} / M$ is the payload fraction of the total satellite mass after a single orbit adjustment maneuver; and

$-C_{e}$ is the specific energy consumption of the satellite correction maneuver. 
Table 3 Laser thruster characteristics for two cases of the satellite velocity decrement

\begin{tabular}{cccccccccc}
\hline $\begin{array}{c}\Delta V, \\
\mathrm{~km}\end{array}$ & $\begin{array}{l}\eta, \\
\%\end{array}$ & $\begin{array}{c}I_{\mathrm{sp}}, \\
\mathrm{s}\end{array}$ & $\begin{array}{c}C_{m}, \\
\text { dyne/W }\end{array}$ & $\begin{array}{c}F_{t}, \\
\mathrm{~N}\end{array}$ & $\begin{array}{l}\dot{m}, \\
\mathrm{~g} / \mathrm{s}\end{array}$ & $\begin{array}{c}P, \\
\mathrm{~kW}\end{array}$ & $m_{\mathrm{pl}} / M$ & $\begin{array}{c}Q, \\
\mathrm{~kJ} / \mathrm{kg}\end{array}$ & $\begin{array}{c}C_{e}, \\
\mathrm{~kJ} / \mathrm{kg}\end{array}$ \\
\hline \multirow{2}{*}{20} & 80 & 598 & 217.8 & 31.8 & 5.4 & 14.6 & 0.993 & 18,787 (Delrin $\left.^{\circledR}\right)$ & 15.1 \\
& 80 & 1000 & 364 & 31.8 & 3.2 & 8.76 & 0.9958 & 57,000 & 9.1 \\
\hline \multirow{2}{*}{100} & 80 & 600 & 219 & 49.7 & 8.4 & 22.7 & 0.989 & 18,787 (Delrin $\left.^{\circledR}\right)$ & 22 \\
& 80 & 1000 & 364 & 49.7 & 5.08 & 13.7 & 0.994 & 57,000 & 13.1 \\
\hline
\end{tabular}

The laser propulsion efficiency is assumed to be maintained at the level of $80 \%$ (see Table 1). The first and the second lines represent the laser propulsion characteristics at specified values of the thrust $F_{t}$, the combustion energy $Q$, and the specific impulse $I_{\mathrm{sp}}$.

As is seen, the use of Delrin ${ }^{\circledR}$ (polyoxymethylene) only as a propellant for the laser thruster cannot provide the necessary characteristics of the thrust which are $I_{\mathrm{sp}} \sim 1000 \mathrm{~s}$ and $C_{m} \sim 100 \mathrm{dyn} / \mathrm{W}$, respectively. To attain these characteristics, a new propellant with the specific combustion heat $Q$ of $57,000 \mathrm{~J} / \mathrm{kg}$ in the order of magnitude should be created. In this case, the specific energy consumption $C_{e}$ of the orbit adjustment maneuver, the laser power, and the stored mass of the propellant will be minimal ones.

\section{CONCLUDING REMARKS}

A laser orbital transfer vehicle as well as a laser thruster can be used to mitigate the problem of space debris accumulation in LEO. An ASLPE operating both in CW and repetitively-pulsed modes is assumed to be used to design a laser propulsion device. To produce a thrust at space conditions efficiently, such power-generating propellant as a polyoxymethylene (Delrin $\left.{ }^{\circledR}\right)$ is suggested to be used. To increase the propulsion efficiency, a new propellant with a combustion energy of $57,000 \mathrm{~kJ} / \mathrm{kg}$ should be created.

\section{REFERENCES}

1. Phipps, C. R., and M. M. Michaelis. 1994. NEO-LISP: Deflecting near-Earth objects using high average power, repetitively pulsed lasers. 23rd European Conference on Laser Interaction with Matter. St. John's College, Oxford, England. LA-UR 943124 .

2. Lobanovsky, Yu. 1996. Concept of an advanced reusable aerospace transportation system. La Recherché Aerospatiale 2. 
3. Contzen, J.P., and J. Muylaert. 2010. Scientific aspects of space debris re-entry. ISTC Workshop on Mitigation of Space Debris. Von Karman Institute for Fluid Dynamics.

4. Launch vehicle energia. http://www.energia.ru/english/energia/launchers/vehicle_ energia-pe.html.

5. Kantrowitz, A. 1972. Propulsion to orbit by ground-based lasers. Aeronautics Astronautics 10(5):40-42.

6. Nebolsine, P.E., and A.N. Pirri. 2002. Laser propulsion: The early years. AIP Conference Proceedings 664:11-21.

7. Rezunkov, Yu. A. 2003. Laser propulsion for LOTV space missions. AIP Conference Proceedings 702:228-40.

8. Phipps, C., M. Birkan, W. Bohn, H. Horisawa, T. Lippert, Yu. A. Rezunkov, A. Sasoh, W. Schall, and J. Sinko. 2010. Laser ablation propulsion. J. Propul. Power 26(4):609-37.

9. Rezunkov, Yu. A. 2011. Efficiency of high-power laser propulsion. Int. J. Aerospace Innovations 3(2):59-75.

10. Ageichik, A. A., E. V. Repina, Yu. A. Rezunkov, and A.L. Safronov. 2009. Detonation of CHO-propellants in a laser propulsion engine. Russ. J. Techn. Phys. 54(3):402-9.

11. Assovskiy, I. G. 2005. Physics of combustion and interior ballistics. M.: Nauka. 360 p. [In Russian.]

12. Liukonen, R. A. 1992. Efficiency of conversion of radiation power into mechanical pulse in the laser propulsion engine. Russ. J. Techn. Phys. 18(7):76-79.

13. Grozdovsky, G.L., Yu. N. Ivanov, and V.V. Tokarev. 1966. Mechanics of space flights of vehicles with a low thrust. M.: Nauka.

14. Next generation space telescope. htlp//ngst.gsfc.nasa.gov.

15. Garrison, P., and G. F. Stoky. 1988. Space rocket engines of the future. J. Propul. Power 6:520-25.

16. Ageichik, A. A., M. S. Egorov, Yu. A. Rezunkov, A. L. Safronov, and V. V. Stepanov. 2003. Aerospace laser propulsion engine. Russ. Patent No. 2266420.

17. Rezunkov, Yu. A., A. L. Safronov, A. A. Ageichik, and M. E. Egorov. 2005. Performance characteristics of laser propulsion engine operating both in $\mathrm{CW}$ and in $\mathrm{RP}$ modes. AIP Conference Proceedings 830:3-13.

18. Rezunkov, Yu. A., M. S. Egorov, S. G. Rebrov, E. V. Repina, and A. L. Safronov. 2009. Laser fine-adjustment thruster for space vehicles. AIP Conference Proceedings 1230:107-17. 